\title{
Attenuated Cyclooxygenase-2 Expression Contributes to Patent Ductus Arteriosus in Preterm Mice
}

\author{
DARSHINI B. TRIVEDI, YUKIHIKO SUGIMOTO, AND CHARLES D. LOFTIN
}

\begin{abstract}
Department of Pharmaceutical Sciences [D.B.T., C.D.L.], College of Pharmacy, University of Kentucky, Lexington, Kentucky 40536; Department of Physiological Chemistry [Y.S.], Faculty of Pharmaceutical Sciences, Kyoto University, Kyoto 606-8501, Japan
\end{abstract}

\begin{abstract}
Patent ductus arteriosus (DA) is the second most common congenital heart defect, the incidence of which is increased in premature infants, although mechanisms responsible are not clear. Our previous studies with genetic or pharmacological inactivation of cyclooxygenase-2 (COX-2) in mice, emphasized the importance of this enzyme in normal DA closure. The current study was designed to determine whether reduced COX-2 expression contributes to patent DA in preterm mice. Real-time PCR analysis indicated that COX-2 expression in the fetal mouse DA significantly increased with advancing gestational age. Furthermore, we observed a significant induction in COX-2 expression in the DA at $3 \mathrm{~h}$ after birth at full-term gestation. In contrast, COX-2 expression was significantly attenuated in the DA of preterm neonatal mice. DA closure was incomplete in preterm mice at $3 \mathrm{~h}$ postpartum, a time-point when the DA of full-term neonates was completely remodeled. Additionally, COX-2 expression was significantly attenuated in the DA of mice deficient in the prostanoid receptor EP4, which also show a patent DA phenotype, suggesting the importance of this receptor for the induction of COX-2 required for DA closure. Overall, these studies suggest that attenuated expression of COX-2 may contribute to increased patent DA at preterm gestation. (Pediatr Res 60: 669-674, 2006)
\end{abstract}

$\mathrm{T}$ he DA is a fetal artery that connects the main pulmonary artery to the descending aorta. A dilated (patent) DA in utero allows greater than $90 \%$ of the right ventricular cardiac output to bypass the high-resistance vascular beds of the uninflated lungs, and thus is vital for fetal health (1). A failure of the DA to close after birth is a condition termed patent DA, which is the second most common congenital heart defect $(2,3)$. Patent DA is more common in premature infants than those born at full term, however, the mechanisms responsible for the increased incidence have not been clearly established.

A number of factors such as nitric oxide, endothelin and oxygen tension have been shown to modulate ductal tone (4). However, the agents primarily responsible for the physiologic regulation of the DA are a family of lipid mediators known as prostanoids, which include PGs and thromboxane $\mathrm{A}_{2}$ (4). The initial reaction in the synthesis of all prostanoids is catalyzed by two isoforms of prostanoid $\mathrm{G} / \mathrm{H}$ synthase, also known as

Received May 12, 2006; accepted August 8, 2006.

Correspondence: Charles D. Loftin, Ph.D., 414 College of Pharmacy, 725 Rose Street, University of Kentucky, Lexington, KY 40536-0082; e-mail: cdloft2@uky.edu

This work was supported by a Scientist Development Grant (0430153N) from the American Heart Association.

DOI: 10.1203/01.pdr.0000246480.13170.c0
COX-1 and COX-2 (5). Individual prostanoids act through specific receptors to mediate their biologic responses.

In numerous species including humans, $\mathrm{PGE}_{2}$ has long been known to be a potent dilator of the DA (4). Traditionally, the predominant function of the COX isoforms expressed in the DA, was thought to be the synthesis of $\mathrm{PGE}_{2}(6,7)$. We have previously reported that genetic deficiency of COX-2 results in patent DA in mice after birth, suggesting the synthesis of contractile prostanoids by COX-2, that is important in normal DA closure $(8,9)$. The current study emphasizes a vital role for the induction of COX-2 in DA closure after full-term birth and suggests that attenuated COX-2 expression at preterm gestation may contribute to increased patent DA.

There are four genes that have been identified which encode the $\mathrm{PGE}_{2}$ receptors, namely EP1, EP2, EP3, and EP4 (10). In mice and other species, it has been known that $\mathrm{PGE}_{2}$ mediates its dilatory actions by activating either EP2, EP3, or EP4 receptors (11). Of the mice deficient in each of the four EP receptors, only EP4-deficient mice exhibit a patent DA phenotype, which is a paradoxical finding given the known dilatory function of this receptor $(12,13)$. Our current study shows that COX-2 expression is attenuated in the DA of EP4-deficient mice, suggesting that an interplay between COX-2 and EP4 is required for initiating normal DA closure after birth.

\section{METHODS}

\begin{abstract}
Animals. The wild-type mice used in these studies (8-10 wk of age) were on a mixed background of C57BL/6 J and 129/Ola and have been maintained by continuous intercrossing with mice from the same colony, as we have reported previously $(8,9)$. EP4 wild-type, heterozygous, and homozygous -deficient mice were generated by crossing EP4 heterozygous mice that were backcrossed 10 times to C57BL/6CrSlc (Japan SLC, Shizuoka, Japan). EP4 mutants were genotyped by PCR as previously described (13). The morning after pairing was designated as gestational d 0.5 upon detection of a copulation plug. Preterm wild-type mice were delivered by cesarean section on gestational d 16 ( $82 \%$ gestation) or 17 (87\% gestation). Full-term wild-type mice were delivered either by cesarean section or natural birth on gestational d 19.5 (100\% gestation). Mice were housed under barrier conditions with food and water provided ad libitum and all experiments were conducted with the IACUC approval at the University of Kentucky.
\end{abstract}

Abbreviations: DA, ductus arteriosus; COX, cyclooxygenase; E, eosin; H, hematoxylin; HPRT, hypoxanthine phospho-ribosyl transferase; PG, prostaglandin 
Tissue collection and RNA isolation. Mice were euthanized in a $\mathrm{CO}_{2}$ chamber followed by thoracotomy, to expose the heart and great vessels. Individual DAs and aortas were dissected without contamination of surrounding vessels. Tissue was individually homogenized in lysis buffer and RNA was isolated using the RNAqueous Micro Kit (Ambion, Austin, TX), which yielded $\sim 150$ ng total RNA per DA.

Quantitative real-time PCR analysis. Gene expression quantitation was performed in a two-step RT-PCR using Superscript II RT (Invitrogen) and Taqman chemistry. Primer/probe assays for COX-1, COX-2, EP2, EP3, EP4, and TP were purchased from Applied Biosystems (Foster City, CA). mRNA encoding the housekeeping gene, HPRT was quantitated for a normalizing control (14). There was no significant difference in HPRT mRNA levels throughout gestation, when compared with other housekeeping genes (data not shown). Analysis was performed using the relative standard curve method. Because the expression analysis for each gene of interest was performed on separate plates, and because the primers for each gene of interest were of different efficiencies, comparison of expression levels between genes of interest was not performed. DA or aorta tissue from 1 pup was considered as an $n$ of 1 and a minimum $n$ of 5 was used for determining statistically significant differences (Mann-Whitney test, Kruskal-Wallis test, or two-way ANOVA).

Histology. As we have described previously $(8,9)$, upper torsos were transected above the rib cage, fixed in $10 \%$ neutral buffered formalin, and processed (paraffin embedded) for histologic analysis. The entire length of the DA from the descending aorta to the bifurcation of the pulmonary arteries was serial sectioned, followed by immunohistochemical analysis for COX-2 (primary antibody from Cayman Chemical, Ann Arbor, MI) or smooth muscle actin (Dako Cytomation, Carpinteria, CA). Antibody binding was detected using the Vectastain Elite ABC kit (Vector Laboratories, Burlingame, CA), and sections were also stained with $\mathrm{H}$ and $\mathrm{E}$ for morphologic analysis of DA closure.

\section{RESULTS}

Attenuated constriction of the DA in preterm fetal mice. We examined the effect of gestational age on indomethacininduced fetal DA constriction in mice. Compared with the completely patent DA of vehicle-treated controls (Fig. 1A), indomethacin treatment induced complete occlusion of the DA of late-term fetal mice on gestational d 18.5 (Fig. 1B). In contrast, indomethacin resulted only in partial constriction of the preterm fetal DA of mice on gestational d 16 (Fig. 1C). These studies show that in mice, the preterm gestation fetal DA is less responsive to pharmacologically-induced constriction, and suggest that near full-term maturation is required for complete development of DA contractile function.

Expression of COX-1 and COX-2 $\mathrm{mRNA}$ in the fetal DA at multiple stages of gestation. We examined the time-course of COX-2 expression in the DA of fetal mice at multiple stages of gestation. To perform these studies, fetuses were obtained by cesarean section on d 16, 17, and 19.5 of gestation, and DA tissue was excised immediately $(0 \mathrm{~h})$ for analysis of mRNA expression. Comparative quantitative analysis of COX-2 mRNA expression showed that COX-2 expression significantly increased with advancing gestation, with the highest expression in the DA on d 19.5 (Fig. 2A). Furthermore, the gestation d 16 time-point with the lowest level of COX-2 mRNA coincided with the time at which we observed incomplete indomethacin-induced constriction of the fetal DA (Fig. 1C). These data demonstrate a correlation between reduced DA constriction and attenuated COX-2 expression in the DA of preterm mice.

We also quantitated COX-1 mRNA expression in the DA at the various stages of gestation. We found that in contrast to COX-2 expression, the expression of COX-1 did not significantly change with advancing gestational age (Fig. 2B). This

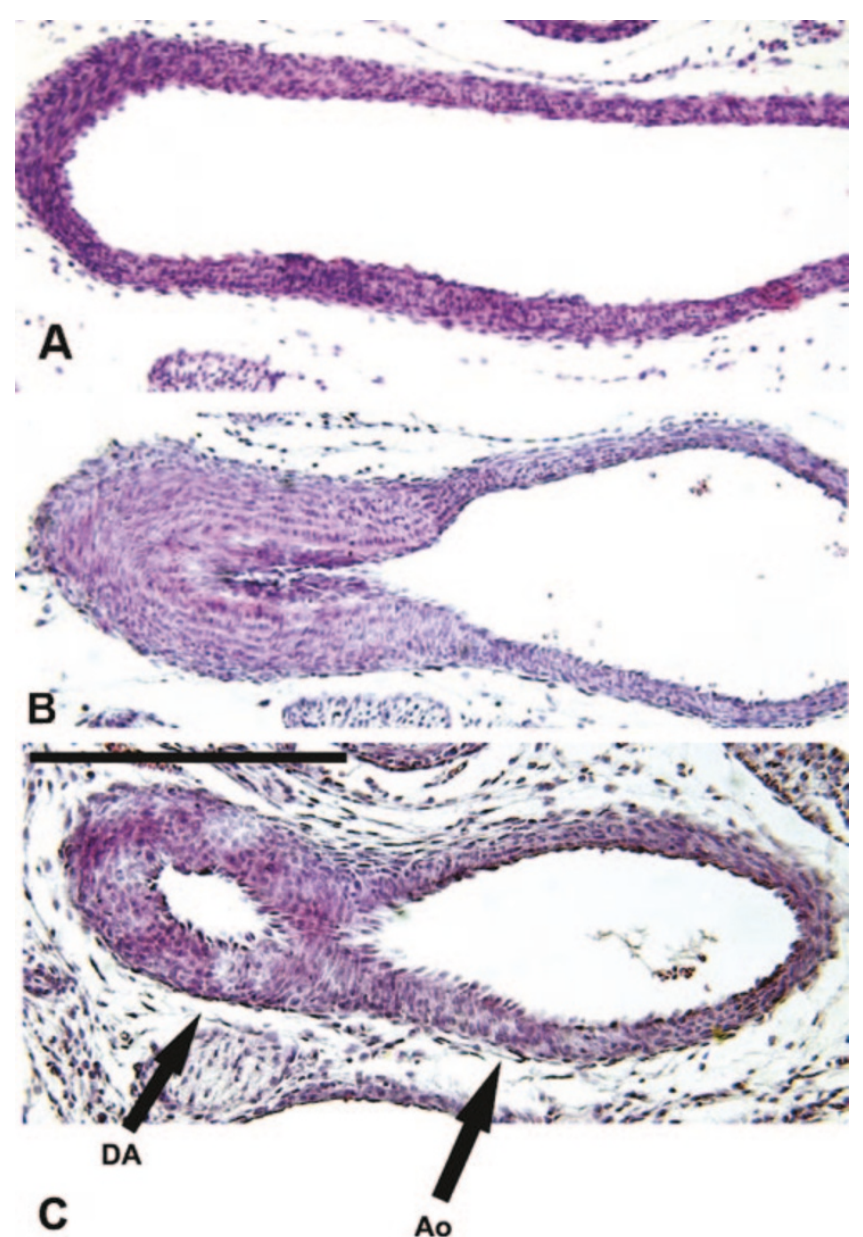

Figure 1. Incomplete indomethacin-induced constriction of the DA in preterm fetal mice. Indomethacin was administered to pregnant dams on gestational d 16 or $18.5(50 \mathrm{mg} / \mathrm{kg}$, oral gavage $5 \%$ gum arabic). Four hours after dosing, fetuses were euthanized for DA analysis. $n \geq 5$ per group. DA of fetal mice treated with $(A)$ vehicle or $(B)$ indomethacin on gestational d 18.5. (C) DA of fetal mice treated with indomethacin on gestational d 16 . Ao, descending aorta; scale bar, $200 \mu \mathrm{m}$. Sections are stained with H\&E.

finding suggests that alterations in COX-1 mRNA expression are not associated with in utero development of fetal DA contractile function.

Postnatal expression of COX-1 and COX-2 in the DA of full-term neonatal mice. We examined COX-2 mRNA expression in the DA of full-term neonates, immediately after birth $(0 \mathrm{~h})$ when the DA was patent, and $3 \mathrm{~h}$ after birth when the DA was completely closed. Quantitative real-time PCR analysis indicated that COX-2 mRNA dramatically increased (approximately 10 fold) in the DA when compared between 0 and $3 \mathrm{~h}$ postpartum on gestational d 19.5 (Fig. 3A). This increase in COX-2 mRNA expression from 0 to $3 \mathrm{~h}$ after birth was unique to the DA, as no such increase in COX-2 expression was observed in the adjacent aorta. We also examined COX-2 protein expression by immunohistochemistry and found that significant COX-2 protein was localized in the smooth muscle cells of the DA with evidence of perinuclear expression, but not in the smooth muscle cells of the adjacent aorta (Fig. $3 B$ ). Therefore, a significant increase in smooth muscle cell expression of COX-2 accompanies closure of the DA after full-term birth. 

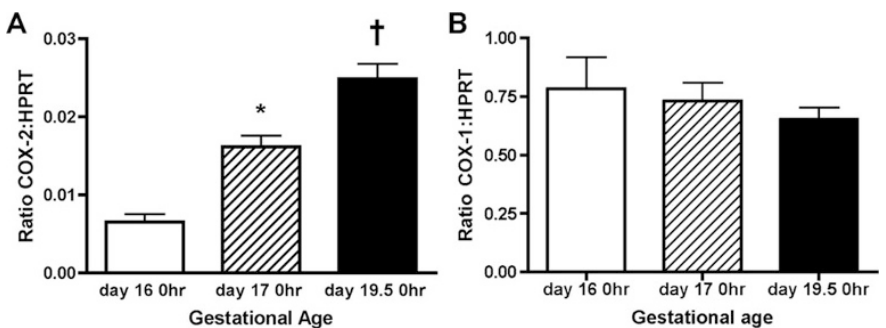

Figure 2. Expression of COX-2, but not COX-1, increases in the fetal DA with advancing gestation. Preterm (gestational d 16 and 17) and full-term (gestational d 19.5) mice were obtained at $0 \mathrm{~h}$ (euthanized immediately after birth) for analysis of mRNA expression for COX-1 or COX-2 in the DA by real-time PCR. The housekeeping gene HPRT was used as an internal control for normalizing mRNA levels. COX-1 and COX-2 mRNA reactions were run on separate plates and arbitrary unit values are not comparable between genes. Data represented as mean $(A)$ COX-2 or $(B)$ COX-1 mRNA levels \pm SEM. *Significantly different from $0 \mathrm{~h}$ on d 16, $p<0.01$ (Kruskal-Wallis test), $n \geq$ 10. $†$ Significantly different from $0 \mathrm{~h}$ on $\mathrm{d} 16$ and $0 \mathrm{~h}$ on d 17, $p<0.001$ (Kruskal-Wallis test), $n \geq 10$.

We also compared COX-1 expression in the full-term neonatal mouse DA between 0 and $3 \mathrm{~h}$ of birth. In contrast to COX-2 expression, the expression of COX-1 mRNA was constitutive with no significant difference between the two time-points (Fig. 3C).

Incomplete postnatal closure of the DA in preterm neonatal mice. The effect of prematurity on DA closure was determined by comparing the DAs of neonates delivered at preterm gestation with those delivered at full-term gestation. Our findings indicate that DA closure was complete in all neonates analyzed at $3 \mathrm{~h}$ after full-term birth on d 19.5 (Fig. $4 A$ ). In contrast, significant lumen was visible in the DAs of
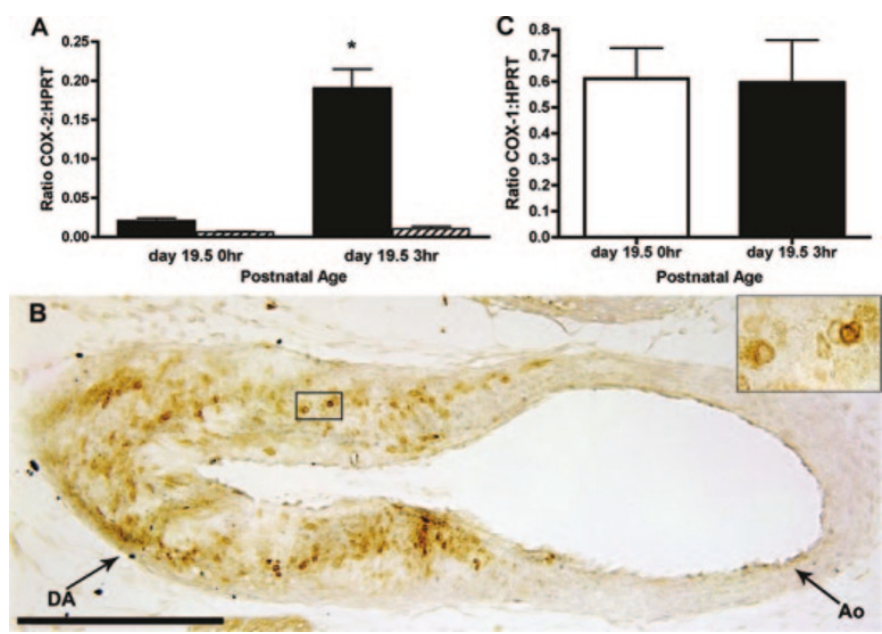

Figure 3. Induction of COX-2 expression in the DA of full-term neonatal mice. (A) Full-term mice were delivered on gestational d 19.5 and euthanized at 0 (immediately after birth) or $3 \mathrm{~h}$ after birth for analysis of COX-2 mRNA expression in the DA (black bars) or aorta (cross-hatched bars) (mean \pm SEM). *Significantly different from $0 \mathrm{~h} \mathrm{DA}$ and $3 \mathrm{~h} \mathrm{Ao} ; p<0.001$ (two-way ANOVA), $n \geq 10$. (B) Immunohistochemical analysis of COX-2 expression. Full-term mice were delivered on gestational d 19.5 and euthanized at 2 or $3 \mathrm{~h}$ postpartum. Brown staining (di-amino benzidine, DAB) indicates COX-2 expression. Figure shown is representative of neonatal DA $2 \mathrm{~h}$ postpartum. Inset shows perinuclear staining. Ao, descending aorta; scale bar, $200 \mu \mathrm{m}$. (C) COX-1 mRNA expression in the DA at 0 and $3 \mathrm{~h}$ after birth on gestational d 19.5 (mean \pm SEM).

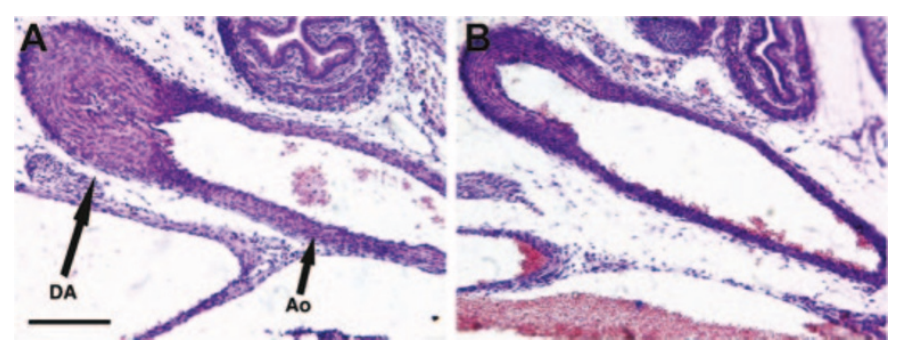

Figure 4. Incomplete closure of the DA in preterm neonatal mice $(n>5$ per group). Preterm gestational d 17 and full-term gestational d 19.5 neonates were euthanized $3 \mathrm{~h}$ postpartum for histologic analysis of DA closure. $(A)$ Day 19.5 neonatal DA; $(B)$ day 17 neonatal DA. Sections are stained with H\&E. Ao, descending aorta; scale bar, $200 \mu \mathrm{m}$.

all preterm neonates that were analyzed at $3 \mathrm{~h}$ postpartum on gestational d 17 (Fig. 4B). These findings indicate that the DAs of neonatal mice born premature show incomplete closure.

Postnatal expression of COX-1 and COX-2 in the DA of preterm neonatal mice. To determine whether the incomplete closure of the DA in preterm neonatal mice that we observed (Fig. 4) was associated with attenuated expression of COX-2, we compared COX-2 expression in the DAs between preterm (d 17) and full-term (d 19.5) neonates at $3 \mathrm{~h}$ after birth. In comparison to the level of expression observed in the DA of full-term neonates, COX-2 mRNA expression in the DA of preterm neonates, was significantly lower (Fig. 5A). The attenuated COX-2 expression coincided with the time-point at which we observed incomplete closure of the DA at preterm
A

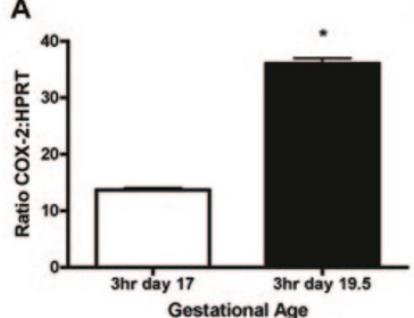

Gestational Age
B

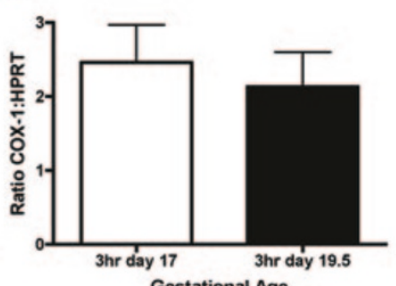

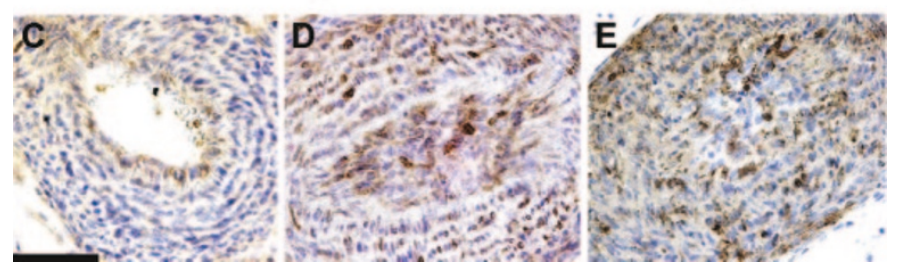

Figure 5. Increased COX-2 mRNA and protein expression in DA smooth muscle of full-term neonatal mice. Preterm (gestational d 17) and full-term (gestational d 19.5) mice were euthanized at $3 \mathrm{~h}$ postpartum. (A) COX-2 mRNA expression is significantly greater in the DA of d 19.5 neonates compared with the DA of d 17 neonates. ${ }^{*} p<0.05$ (Mann-Whitney test), $n$ $\geq 5$. (B) COX-1 mRNA expression is not significantly different between the DA of preterm and full-term neonatal mice. Data are represented as mean COX-1 or COX-2 mRNA levels \pm SEM. (C) The DA of a d 17 neonatal mouse at $3 \mathrm{~h}$ after birth shows significantly fewer COX-2-positive cells compared with $(D)$ the DA of a d 19.5 neonatal mouse at $3 \mathrm{~h}$ postpartum. Significant lumen is visible in $(C)$, indicative of partial DA closure due to prematurity. (E) DA of a d 19.5 neonatal mouse at $3 \mathrm{~h}$ after birth, stained for smooth muscle actin. Brown staining (DAB reagent) indicates COX-2 or actin expression and sections are counterstained with hematoxylin (blue). Figures shown (magnification, $\times 300$ ) are representative of immunohistochemical analysis of neonatal DA from a minimum of five mice. Scale bar, $50 \mu \mathrm{m}$. 

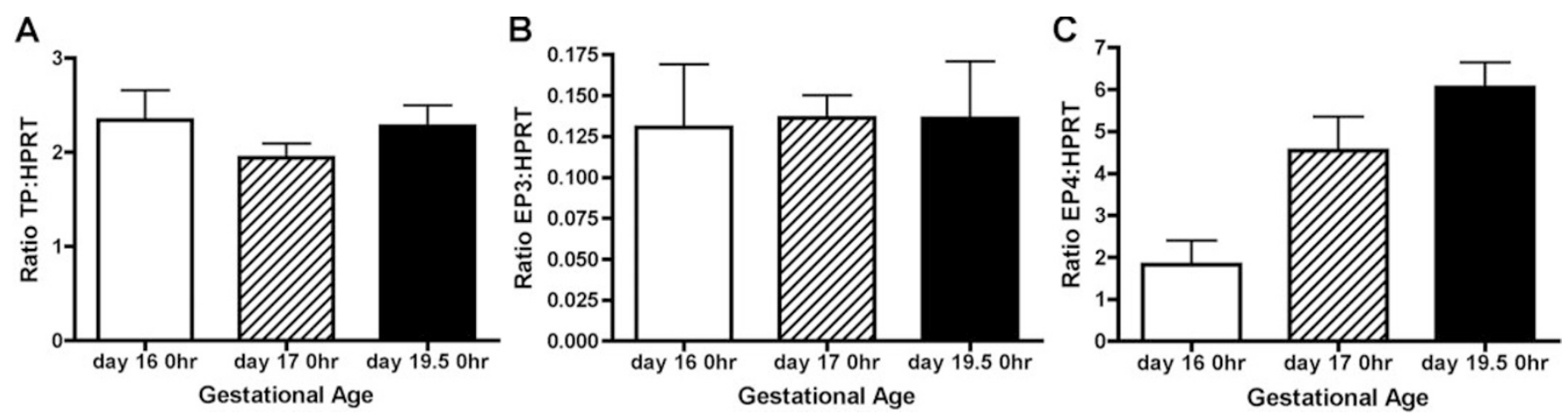

Figure 6. Expression of EP4, but not EP3 or TP, increases with advancing gestational age. Preterm (gestational d 16 and 17) and full-term (gestational d 19.5) mice were obtained at $0 \mathrm{~h}$ for analysis of mRNA expression in the DA. Data represented as mean $(A) \mathrm{TP},(B) \mathrm{EP} 3$, and $(C)$ EP4 mRNA levels \pm SEM. EP4 mRNA expression in the DA significantly increased throughout gestation, $p<0.05$ (Kruskal-Wallis test), $n \geq 8$.

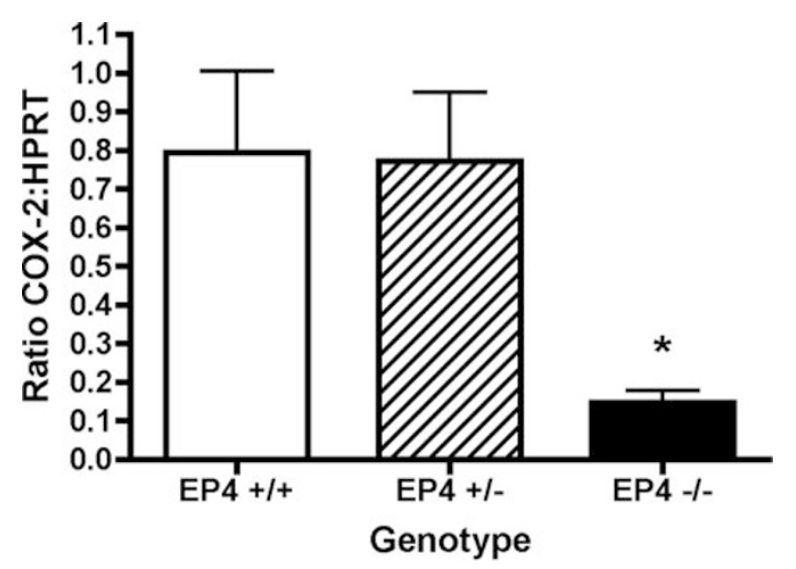

Figure 7. Reduced COX-2 expression in the DA of EP4-deficient neonatal mice. Full-term gestation (d 19.5) EP4 wild-type $\left(E P 4^{+/+}\right)$, heterozygous $\left(E P 4^{+/-}\right)$and homozygous-deficient $\left(E P 4^{--}\right)$mice were euthanized $3 \mathrm{~h}$ after birth for analysis of COX-2 expression in the DA. Data represented as mean COX-2 mRNA levels \pm SEM. *Significantly different from $E P 4^{+/+}$and $E P 4^{+/-}$DA, $p<$ 0.01 (Kruskal-Wallis test), $n \geq 8$.

gestation (Fig. 4B). In contrast to COX-2 expression, there was no significant difference in COX-1 mRNA expression between DAs from preterm and full-term neonatal mice at $3 \mathrm{~h}$ postpartum (Fig. 5B). Immunohistochemical analysis of COX-2 protein expression indicated that the DA of full-term neonatal mice showed significantly greater number of COX2-positive cells than the DA of preterm neonatal mice (Fig. 5, $C$ and $D$ ). Furthermore, the COX-2 expression was primarily localized in the smooth muscle cells of the DA (Fig. 5E). Thus, these data establish a direct correlation between the attenuated COX-2 expression and increased patent DA.

Quantitation of prostanoid receptors in the fetal DA at multiple stages of gestation. Previous reports have suggested that the prostanoid receptors EP2, EP3, EP4, and TP may be involved in modulating DA tone $(9,11,15,16)$. In the current studies, we examined mRNA expression levels for these prostanoid receptors. As shown in Figure 6A, the fetal mouse DA expressed a significant level of TP, however, the level of expression did not significantly change throughout gestation. EP2 mRNA expression was nearly undetectable in the fetal DA (data not shown). The expression of EP3 mRNA was constitutive in the fetal DA, when examined on d 16, 17, and
19.5 of gestation (Fig. $6 B$ ). In contrast, expression of the EP4 receptor in the fetal mouse DA increased significantly from gestational d 16 to 19.5 (Fig. 6C).

Reduced COX-2 mRNA expression in the DA of EP4deficient neonatal mice. In addition to the COX-deficient mice, mice deficient in the $\mathrm{PGE}_{2}$ receptor EP4 also exhibit a patent DA phenotype $(12,13)$. This finding is surprising because it is well established that $\mathrm{PGE}_{2}$ produces dilation of the DA (4). Furthermore, there is no evidence that EP4 receptor activation directly induces constriction of the smooth muscle of the DA or any other vasculature. We hypothesized that the expression of EP4 may be required for the induction of COX-2, important for normal DA closure. We therefore compared COX-2 expression in the DA of EP4-deficient and EP4-expressing neonatal mice. Our results indicate that COX-2 expression is significantly attenuated in the DAs of EP4-deficient mice at $3 \mathrm{~h}$ after birth, compared with the DAs of littermate EP4 heterozygous or wild-type mice (Fig. 7).

\section{DISCUSSION}

In humans, patent DA is more common in premature infants, than those born at full-term, although mechanisms responsible for the increased incidence are poorly understood. We have previously reported that genetic or pharmacological inactivation of COX-2 results in patent DA in mice, indicating the importance of COX-2 in postnatal closure of the DA $(8,9)$. The current studies were aimed at better understanding the COX-2-dependent mechanisms of DA closure and elucidating the factors responsible for patent DA at preterm gestation. Our findings are the first to suggest that reduced expression of COX-2 in the DA at preterm gestation contributes to increased patent DA.

Fetal DA constriction induced by the nonselective COX inhibitor indomethacin has long been used to study pharmacologically induced contractile responses in the DA (17). Maternal administration of indomethacin results in premature constriction of the DA by inhibiting the synthesis of prostanoids important for DA dilation (4). We have reported that similar to previous studies with indomethacin, acute administration of COX-2-selective inhibitors results in premature DA constriction, whereas chronic administration of COX-2 inhibitors results in postnatal patent DA in mice (9). A more recent 
report replicated these studies and showed similar results (18). These contrasting effects suggest opposing actions of different prostanoids on the regulation of DA tone. However, mechanisms by which acute indomethacin administration results in DA constriction in utero, without affecting postnatal DA closure, remain to be fully elucidated. Nevertheless, acute maternal administration of indomethacin is an effective functional marker for fetal DA constriction.

Previous studies in rats and humans have shown that the preterm fetal DA is less responsive to indomethacin-induced constriction, in contrast to the DA of full-term fetuses $(19,20)$. In the current studies, we examined the effect of gestational age on indomethacin-induced fetal DA constriction in mice. Our results show that the preterm fetal DA on gestational d 16 is less responsive to the constricting effects of indomethacin, compared with the late gestation fetal DA on $d$ 18.5. Therefore, in mice the contractile nature of the DA develops late in gestation.

We have previously reported that the DA of COX-2deficient fetuses fails to constrict in response to indomethacin, suggesting the importance of COX-2 expression for in utero contractile function (8). Therefore, in the current study, we investigated the relationship between the ontogeny of COX-2 expression and the developmental regulation of fetal DA constriction. COX-2 expression in the DA was significantly greater in full-term fetuses than that in preterm fetuses. Our previous report, together with our current study suggest that the contractile function of the DA that develops toward the end of term requires the in utero increase in COX-2 expression with advancing gestational age.

Normal DA closure in mice at full-term gestation is complete within $3 \mathrm{~h}$ after birth. With our finding that incomplete development resulted in attenuated fetal DA constriction, we investigated whether prematurity in gestation also compromised normal DA closure after birth. We observed that at $3 \mathrm{~h}$ postpartum, DA closure was incomplete in preterm neonates, in contrast to the completely remodeled DA of $3 \mathrm{~h}$-old neonates born at full-term gestation. This suggests that similar to humans, premature birth compromises postnatal DA closure in mice.

The factors responsible for inducing normal closure of the DA after birth have not been clearly defined. Our previous studies using mice deficient in COX-2, clearly demonstrate the importance of this enzyme in postnatal DA closure (8). In mice, DA closure commences within $30 \mathrm{~min}$ after full-term birth. Our current studies show that COX-2 expression is dramatically induced in the neonatal DA within $3 \mathrm{~h}$ of birth at full-term gestation, which correlates with the time-point when the DA is completely remodeled. This suggests that induction of COX-2 expression in the DA may be required for achieving permanent closure after full-term birth. Therefore, we hypothesized that the incomplete closure of the DA in preterm gestation neonatal mice that we observed, may result from attenuated expression of COX-2. In support of this hypothesis, we found that COX-2 expression was significantly lower in the DAs of neonatal mice delivered at preterm gestation, compared with that expressed in the DAs of full-term neonates. The reduced COX-2 expression corresponded with the time-point at which we observed incomplete closure of the preterm neonatal DA. Thus, our studies demonstrate a direct correlation between the attenuated expression of COX-2 and the failure of complete closure of the DA at preterm gestation.

Previous studies examining developmental regulation of the COX isoforms in the DA have focused on the action of these enzymes in DA dilation. In other species, it has been reported that COX-1 is the predominant isoform expressed in the premature fetal DA, whereas COX-2 is primarily expressed in the DA late in gestation $(6,21)$. Our current findings in mice are in agreement with previous reports, and show that COX-1 is constitutively expressed in the DA throughout gestation, and COX-2 is induced in the DA from preterm to full-term gestation. It has also been suggested that the DA at preterm gestation is more sensitive to dilation in response to $\mathrm{PGE}_{2}$ derived from the activity of $\mathrm{COX}-1$, a process that may contribute to increased postnatal patent DA $(6,11)$. Our work emphasizes the importance of COX-2 in mediating DA closure. Because of its potent dilatory effect, it is unlikely that $\mathrm{PGE}_{2}$ is the COX-2-derived prostanoid that induces DA closure. We propose that COX-2 expressed in the DA synthesizes a prostanoid other than $\mathrm{PGE}_{2}$, which directly mediates DA closure. Therefore, patent DA at preterm gestation may result from constitutive expression of COX-1 together with the attenuated expression of COX-2.

Because $\mathrm{PGE}_{2}$ is a known potent dilator of the DA, several studies in numerous species have focused on examining EP receptor expression in the DA to better understand the role of $\mathrm{PGE}_{2}$ in modulating DA tone. Depending on the species, gene expression in the DA for EP2, EP3, and EP4 does not change, decreases, or increases with advancing gestation $(11,15,16)$. Of the EP receptors that we examined in our current studies, only EP4 mRNA expression significantly increased with advancing gestation. Furthermore, the gestational expression pattern of EP4 mimicked that which we observed for COX-2. The co-regulation of EP4 and COX-2 suggests a relationship between this prostanoid receptor and COX-2, for inducing DA closure.

In addition to the COX-2-deficient mice, mice deficient in the EP4 receptor also exhibit a patent DA phenotype $(12,13)$. This finding is surprising because it is well established that $\mathrm{PGE}_{2}$ produces dilation of the DA. The proposed mechanism for patent DA in the EP4-deficient mice was that the loss of EP4 results in persistent levels of cAMP, which maintain DA dilation (12). A recent report has shown that cAMP levels are increased in the premature ovine DA, making the DA more sensitive to dilation (11). Based on these previous reports, it is possible that the patent DA we observe at preterm gestation in mice, may be due to reduced levels of EP4 and resultant increased levels of cAMP, in addition to attenuated expression of COX-2.

EP4 activation is known to induce COX-2 expression in multiple cell types $(22,23)$. Our studies indicate that the genetic deficiency of EP4 results in attenuated COX-2 expression in the DA and COX-2 is important for DA closure. Thus, our results provide an explanation for the paradoxical finding of patent DA in the EP4-deficient mice, and suggest that EP4 expression may be important for modulating the normal in- 
duction of COX-2 and resultant DA closure after full-term birth.

Acknowledgments. The authors thank Dr. Robert Langenbach (National Institutes of Health/National Institute of Environmental Health Sciences, Research Triangle Park, NC) for providing the breeder pairs of COX-2 wild-type mice and Drs. Shuh Narumiya (Kyoto University, Kyoto, Japan) and Takayuki Maruyama (Ono Pharmaceuticals, Osaka, Japan) for the EP4 heterozygous-deficient breeding pairs.

\section{REFERENCES}

1. Clyman RI, Hardy P, Waleh N, Chen YQ, Maury F, Fouron JC, Chemtob S 1999 Cyclooxygenase- 2 plays a significant role in regulating the tone of the fetal lamb ductus arteriosus. Am J Physiol 276:R913-R921

2. Mani A, Meraji SM, Houshyar R, Radhakrishnan J, Ahangar M, Rezaie TM, Taghavinejad MA, Broumand B, Zhao H, Nelson-Williams C, Lifton RP 2002 Finding genetic contributions to sporadic disease: a recessive locus at $12 \mathrm{q} 24$ commonly contributes to patent ductus arteriosus. Proc Natl Acad Sci U S A 99:15054-15059

3. Mitchell SC, Korones SB, Berendes HW 1971 Congenital heart disease in 56,109 births. Incidence and natural history. Circulation 43:323-332

4. Smith GC 1998 The pharmacology of the ductus arteriosus. Pharmacol Rev 50:35-58

5. Smith WL, DeWitt DL 1996 Prostaglandin endoperoxide H synthases-1 and -2. Adv Immunol 62:167-215

6. Guerguerian AM, Hardy P, Bhattacharya M, Olley P, Clyman RI, Fouron JC, Chemtob S 1998 Expression of cyclooxygenases in ductus arteriosus of fetal and newborn pigs. Am J Obstet Gynecol 179:1618-1626

7. Coceani F, Ackerley C, Seidlitz E, Kelsey L 2001 Function of cyclo-oxygenase-1 and cyclo-oxygenase-2 in the ductus arteriosus from fetal lamb: differential development and change by oxygen and endotoxin. Br J Pharmacol 132:241-251

8. Loftin CD, Trivedi DB, Tiano HF, Clark JA, Lee CA, Epstein JA, Morham SG, Breyer MD, Nguyen M, Hawkins BM, Goulet JL, Smithies O, Koller BH, Langenbach R 2001 Failure of ductus arteriosus closure and remodeling in neonatal mice deficient in cyclooxygenase-1 and cyclooxygenase-2. Proc Natl Acad Sci U S A 98:1059-1064

9. Loftin CD, Trivedi DB, Langenbach R 2002 Cyclooxygenase-1-selective inhibition prolongs gestation in mice without adverse effects on the ductus arteriosus. J Clin Invest 110:549-557

10. Narumiya S, Sugimoto Y, Ushikubi F 1999 Prostanoid receptors: structures, properties, and functions. Physiol Rev 79:1193-1226
11. Waleh N, Kajino H, Marrache AM, Ginzinger D, Roman C, Seidner SR, Moss TJ, Fouron JC, Vazquez-Tello A, Chemtob S, Clyman RI 2004 Prostaglandin E2mediated relaxation of the ductus arteriosus: effects of gestational age on g proteincoupled receptor expression, signaling, and vasomotor control. Circulation 110:2326-2332

12. Nguyen M, Camenisch T, Snouwaert JN, Hicks E, Coffman TM, Anderson PA, Malouf NN, Koller BH 1997 The prostaglandin receptor EP4 triggers remodelling of the cardiovascular system at birth. Nature 390:78-81

13. Segi E, Sugimoto Y, Yamasaki A, Aze Y, Oida H, Nishimura T, Murata T, Matsuoka T, Ushikubi F, Hirose M, Tanaka T, Yoshida N, Narumiya S, Ichikawa A 1998 Patent ductus arteriosus and neonatal death in prostaglandin receptor EP4-deficient mice. Biochem Biophys Res Commun 246:7-12

14. French MC, Littlejohn RP, Greer GJ, Bain WE, McEwan JC, Tisdall DJ 2006 Growth hormone and ghrelin receptor genes are differentially expressed between genetically lean and fat selection lines of sheep. J Anim Sci 84:324-331

15. Smith GC, Wu WX, Nijland MJ, Koenen SV, Nathanielsz PW 2001 Effect of gestational age, corticosteroids, and birth on expression of prostanoid EP receptor genes in lamb and baboon ductus arteriosus. J Cardiovasc Pharmacol 37:697-704

16. Bhattacharya M, Asselin P, Hardy P, Guerguerian AM, Shichi H, Hou X, Varma DR, Bouayad A, Fouron JC, Clyman RI, Chemtob S 1999 Developmental changes in prostaglandin E-2 receptor subtypes in porcine ductus arteriosus-possible contribution in altered responsiveness to prostaglandin E-2. Circulation 100: $1751-1756$

17. Smith GC, McGrath JC 1988 Indomethacin, but not oxygen tension, affects the sensitivity of isolated neonatal rabbit ductus arteriosus, but not aorta, to noradrenaline. Cardiovasc Res 22:910-915

18. Reese J, Anderson JD, Brown N, Roman C, Clyman RI 2006 Inhibition of Cyclooxygenase isoforms in late- but not mid-gestation decreases contractility of the ductus arteriosus and prevents postnatal closure in mice. Am J Physiol Regul Integr Comp Physiol doi: 10.1152/ajpregu. 00259.2006 (accessed August 30, 2006)

19. Moise KJ 1993 Effect of advancing gestational age on the frequency of fetal ductal constriction in association with maternal indomethacin use. Am J Obstet Gynecol 168:1350-1353

20. Momma K, Takao A 1987 In vivo constriction of the ductus arteriosus by nonsteroidal antiinflammatory drugs in near-term and preterm fetal rats. Pediatr Res 22:567-572

21. Bouayad A, Fouron JC, Hou X, Beauchamp M, Quiniou C, Abran D, Peri K, Clyman RI, Varma DR, Chemtob S 2004 Developmental regulation of prostaglandin E2 synthase in porcine ductus arteriosus. Am J Physiol Regul Integr Comp Physiol 286:R903-R909

22. Regan JW 2003 EP2 and EP4 prostanoid receptor signaling. Life Sci 74:143-153

23. Bradbury DA, Newton R, Zhu YM, El-Haroun H, Corbett L, Knox AJ 2003 Cyclooxygenase- 2 induction by bradykinin in human pulmonary artery smooth muscle cells is mediated by the cyclic AMP response element through a nove autocrine loop involving endogenous prostaglandin E2, E-prostanoid 2 (EP2), and EP4 receptors. J Biol Chem 278:49954-49964 